\title{
Aspecto clínico, patológico y terapéutico del Liquen Ampollar
}

Clinical, pathological and therapeutic aspects of bullous lichen

María Mercedes González ${ }^{1 凶}$, Carlos Alejandro Rosales ${ }^{2 \otimes}$, Evelin Elizabeth Barrios ${ }^{2 凶}$, Facundo Cuenya $^{2 \otimes}$, Estefanía Raquel Fernández ${ }^{2 \otimes}$

1. Doctora en Odontología. Profesora Titular por Concurso Cátedra Clínica Estomatológica. Facultad de Odontología de la Universidad Nacional del

Nordeste. Corrientes. Argentina.

2. Odontólogo. Facultad de Odontología de la Universidad Nacional del Nordeste. Corrientes. Argentina.

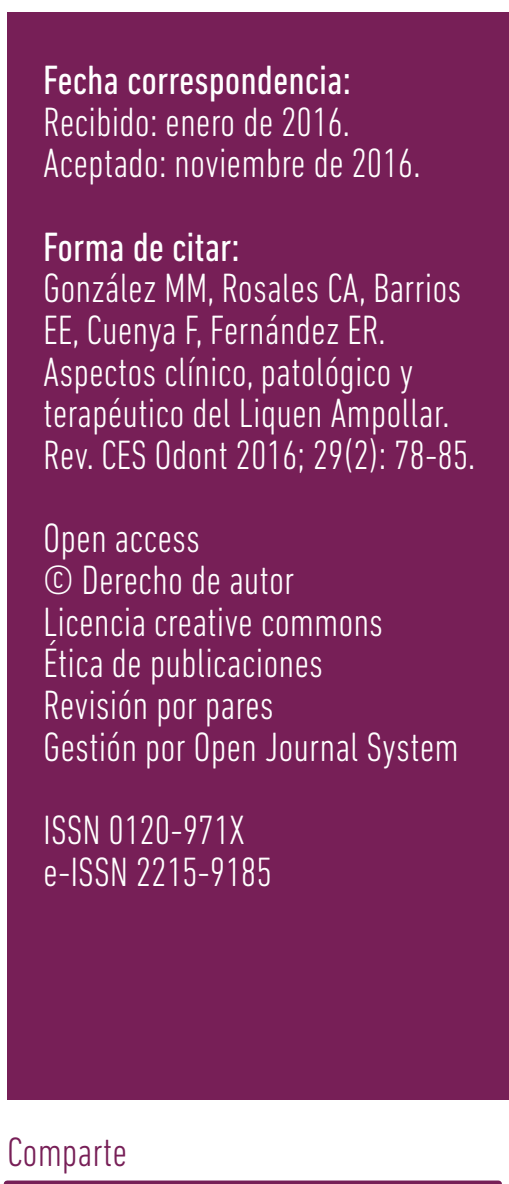

FอG $898 \mathbb{R}^{\circ}$ 国

\section{Resumen}

El liquen plano oral es una enfermedad crónica mucocutánea de etiología autoinmune que afecta principalmente a mujeres entre 40 y 50 años de edad. Una de sus variantes clínicas e histopatológicas se denomina liquen ampollar, considerado como liquen atípico. Clínicamente se manifiesta como ampollas que se rompen fácilmente causando excesivo dolor. Se presenta a la consulta una paciente de 60 años refiriendo dolor urente en fondo de surco próximo a molares inferiores del lado derecho y en el lado izquierdo en la zona de molares superiores. Manifestó ardor en la superficie lingual causándole dificultad para hablar y alimentarse. Presentaba lesiones blancas y rojas extendidas por toda la mucosa bucal. El estudio clínico e histopatológico a través de biopsia constató la presencia de un liquen ampollar. Se inició el tratamiento obteniéndose resultados satisfactorios en las primeras semanas y se indicó iniciar un tratamiento multidisciplinario con dermatólogos y psicoanalistas.

Palabras clave: Liquen plano buloso, liquen plano ampollar, liquen atípico, enfermedad autoinmune, mucosa bucal.

\section{Abstract}

Lichen planus is a chronic mucocutaneous disease of self-immunological etiology that primarily affects women between 40 and 50 years old. One of its clinical and histopathologic variants is called bullous lichen planus, considered an atypical lichen. Clinically it manifests as blisters that break easily causing excessive pain. A 60 year-old female patient attended medical consultation with a main complain of burning pain in the vestibular sulcus next to right lower and left upper molars. The burning sensation on the lingual aspect caused difficulties during speaking or eating, as referred by the patient in the anamnesis. Accompaning white and red lesions spread throughout the oral mucosa. Both clinical examination and histopathological biopsy confirmed the pathological findings. A multidisciplinary treatment approach -with dermatologist and pschycoanalist- was established in the following weeks with satisfactory results.

Keywords: Bullous lichen planus, atypical lichen, autoimmune disease, oral mucosa. 


\section{Introducción}

El liquen plano oral (LPO) es una enfermedad inflamatoria crónica de etiología desconocida, con fisiopatología probablemente de tipo autoinmune mediada por linfocitos T. Existe una predisposición genética dada la presencia de diferentes antígenos de histocompatibilidad y la afectación de varios miembros de una misma familia. Se piensa que la influencia psicosomática puede desencadenar la enfermedad y las recidivas (1). Afecta principalmente a individuos del sexo femenino entre 40 y 50 años de edad (2). Se manifiesta en regiones de trauma frecuente, principalmente en el tercio posterior de la mucosa yugal en un $90 \%$ (3). En menor grado, se presenta en el dorso de la lengua, encía, mucosa labial y bermellón del labio inferior. Las lesiones en paladar, piso de la boca y labio superior no son frecuentes.

La lesión inicialmente se desarrolla en las células basales del epitelio, siendo el infiltrado dérmico una manifestación secundaria. Sin embargo, todavía no ha podido ser demostrada la causa inicial que produce esta alteración en las células epiteliales (4). Histopatológicamente, el liquen plano oral muestra la presencia de hiperqueratosis ortoqueratinizada o paraqueratinizada, la cual se observa clínicamente como pápulas, estrías o placas, y en otras ocasiones se ve atrofia o ulceración epitelial que corresponde clínicamente a zonas rojas. En el tejido conectivo subyacente a las lesiones se ve una banda oscura bien definida, conformada por células inflamatorias en especial linfocitos e histiocitos, se observa también degeneración hidrópica de la capa basal epitelial formando cuerpos coloides o de Civatte y ausencia de displasia epitelial (ㅁ). Al no evidenciarse presencia de anticuerpos, la marcación con inmunofluorescencia es negativa. Por ello debe diferenciarse de entidades como: penfigoides, eritema multiforme e infección con virus herpes simple (9).

Puede afectar piel, uñas, cuero cabelludo (causando alopecia), glande, vulva, vagina, esófago, conjuntiva y membranas de mucosas orales $(\underline{5}, \underline{6})$. No se trata de una infección, su curso evolutivo es benigno aunque en ocasiones puede llegar a sufrir una degeneración maligna (4). Sus manifestaciones bucales comprenden diferentes formas clínicas, que pueden ser diferenciadas en típicas y atípicas. La forma típica es el liquen reticular, que puede adoptar una distribución dendrítica, clásicamente descrito con aspecto de nervaduras de hojas, papular, y/o anular. Estas lesiones se observan preferentemente en la zona posterior de la mucosa yugal de forma bilateral, y con escasa o ninguna sintomatología. Las formas denominadas atípicas, pueden ser la evolución de un liquen típico o primitivas. Se diferencian entre ellas: el liquen queratósico o en placa (variante del liquen reticular); el liquen erosivo o ulcerativo (que se presenta como gingivitis descamativa en la encia adherida); el liquen atrófico (localización preferente en la lengua); y la forma ampollar que puede preceder al erosivo (7). Según el orden de severidad e infrecuencia de aparición clínica, de menor a mayor se pueden mencionar el liquen papular, en placa, atrófico y erosivo; y en muy raras ocasiones el liquen plano ampollar (8).

El liquen plano ampollar es la variable más rara de los tipos de liquen plano oral. Se manifiesta como ampollas que se rompen fácilmente, pueden variar de tamaño (desde unos pocos milímetros hasta varios centímetros), y a menudo se acompañan de sensación de ardor y dolor. Estan resultan de la licuefacción y vacuolización de la capa epitelial basal y se observa comúnmente en la mucosa bucal, especialmente en las áreas posteroinferiores adyacentes al segundo y tercer molar. El segundo sitio más frecuente de manifestación son los bordes laterales de la lengua, mientras que raramente se ve en encía y labios (9). 
Para obtener un diagnóstico acertado se debe realizar una correcta anamnesis y exploración clínica, una biopsia para el estudio histopatológico y un análisis sanguíneo buscando una posible relación con determinados procesos sistémicos. Se recomienda realizar una inmunofluorescencia directa para diagnóstico diferencial y en ocasiones se debe realizar un análisis estructural y otras pruebas diagnósticas (4). La biopsia se recomienda para realizar diagnóstico diferencial temprano entre liquen plano oral y una displasia liquenoide, para la cual esta recomendado manejo oncológico (므).

Su remisión espontánea es poco común y actualmente no hay un tratamiento específico para el liquen plano oral, debido al difícil manejo de estos pacientes por la naturaleza crónica de la enfermedad, con diferentes periodos de actividad y remisión $(\underline{6}, \underline{10})$. Si las lesiones son asintomáticas, por lo general no se realiza tratamiento, sino que se advierte al paciente que debe regresar regularmente para revisión, mínimo cada año o antes si manifiestan síntomas. El tratamiento tiene como objetivo eliminar las ulceraciones, aliviar los síntomas y reducir el riesgo de una posible malignización. Se debe priorizar la eliminación de los factores traumáticos próximos a la lesión, tales como restos radiculares, aristas cortantes, ganchos de prótesis, entre otros. Es necesario también eliminar los factores irritantes locales como el tabaco, alcohol, entre otros. La higiene bucal debe ser exhaustiva eliminando los depósitos de sarro y tratando de reequilibrar psicológicamente al paciente (10). La aplicación tópica de corticoesteroides puede ser de ayuda para el tratamiento inicial y para la terapia de mantenimiento. Puede administrarse como ungüento, pasta, pastillas, enjuague o spray (6). El pronóstico del liquen plano ampollar es satisfactorio en cuanto a la vida del paciente siempre que se lo diagnostique de manera temprana, caso contrario puede producirse una transformación maligna. Evoluciona por brotes y cura en un tiempo promedio de 2 a 6 meses; existen casos cuya evolución se hace crónica y evoluciona en años. Se debe recordar que las lesiones en las mucosas curan más lentamente que las cutáneas (11). El siguiente caso clínico ilustra el diagnostico y manejo de una paciente con liquen plano ampollar.

\section{Presentación de caso clínico}

Acudió al Servicio de Estomatología de la Facultad de Odontología una paciente de sexo femenino de 60 años de edad, quien presentaba dolor urente en la zona de molares superiores izquierda y zona de molares inferiores derecha, donde tenía colocados tres implantes dentarios con sus respectivas coronas. Manifestó también ardor en la superficie lingual. Relató que esta sensación quemante se inició desde la colocación de las coronas sobre implantes en el maxilar inferior hace aproximadamente un año. Manifestó que las molestias se exacerbaban durante cinco a siete días y luego remitían. Padecía este tipo de sintomatología en la mucosa yugal de ambos lados, dos y hasta tres veces en el mes.

En el interrogatorio la paciente manifestó que no fumaba, no se encontraba bajo tratamiento farmacológico, no había sido hospitalizada en los últimos dos años y nunca presentó reacción alérgica a algún medicamento o sustancia. En el examen extraoral se observó ausencia de asimetría facial y adenopatías cervicales. En el examen intraoral se evidenció en la porción central de la cara dorsal de la lengua placas asintomáticas, blancas, circulares, regulares y con bordes definidos que no se desprendían con el raspaje (Figura 1). 


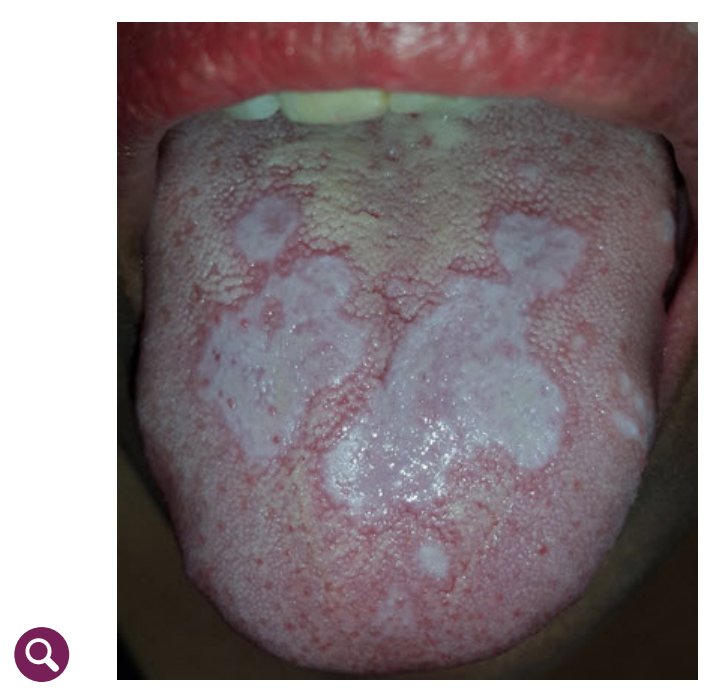

Figura 1. En la porción central de la cara dorsal de la lengua se observan tres placas asintomáticas, blancas, circulares, irregulares, con bordes definidos, de hasta $3 \mathrm{~cm}$ de diámetro que no desprenden con el raspaje.

En las mucosas yugales bilaterales se observó una lesión en forma de trama con aspecto comparable con una red, levemente sobreelevada y simétrica (Figura 2). A nivel de fondo de surco vestibular inferior próximo a tres coronas sobre implantes de las piezas dentarias 44, 45 y 46 se observó una lesión erosiva de gran extensión, aproximadamente de $3 \mathrm{~cm}$ de diámetro con bordes difusos, que producían a la paciente estomatopirosis y disfagia aproximadamente siete días consecutivos (Figura 3). En el fondo de surco vestibular superior del lado izquierdo próximo a la pieza dentaria 26 se evidenciaba una lesión con características similares a la ya descrita con sintomatología dolorosa que se exacerba al ingerir alimentos calientes y/o picantes. Se encontraba comprometido el margen gingival desde la pieza dentaria 21 hasta la 26 inclusive, presentando una gingivitis marginal crónica (Figura 4).

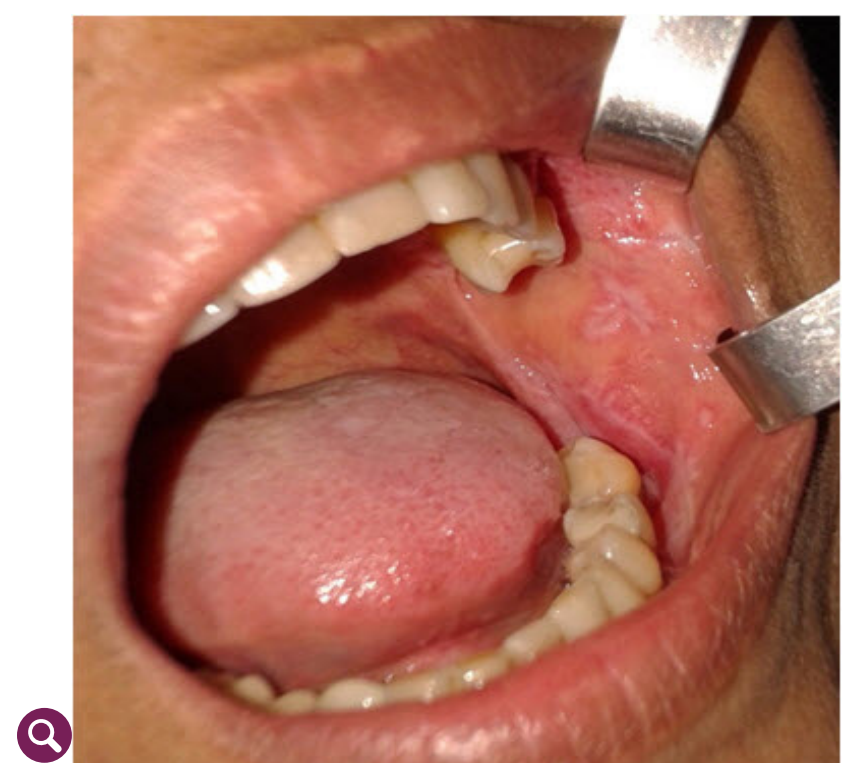

Figura 2. En la mucosa yugal del lado derecho se observan estrías blanquecinas en forma de trama, con relieve rugoso y borde eritematoso. En el fondo del vestíbulo superior próximo a la pieza dentaria 26 se observa una placa blanca con puntillado eritematoso.
Q

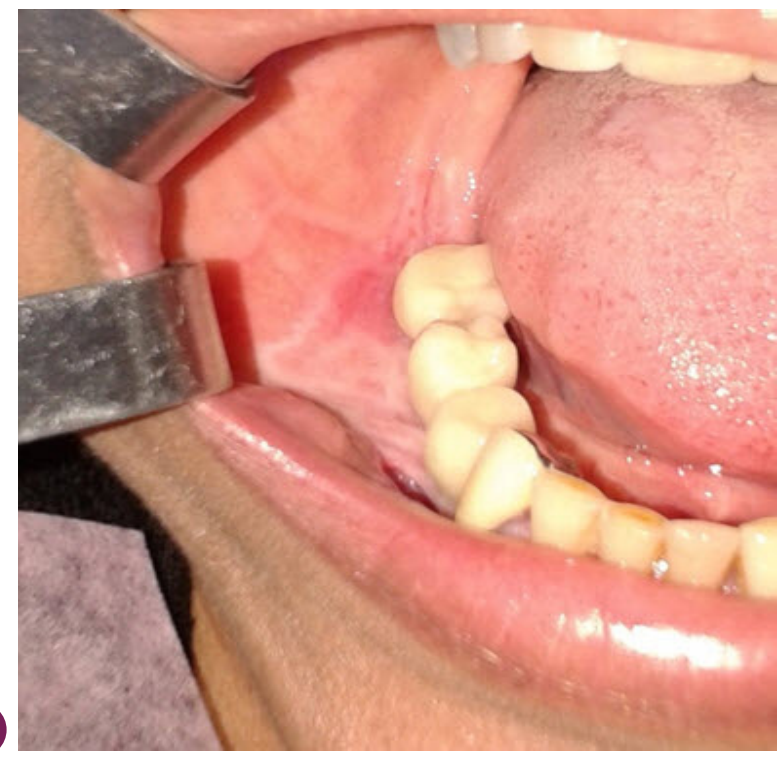

Figura 3. Lesión heterogénea representada por estrías blanquecinas y ulceración rojiza extendida en el fondo de surco vestibular inferior desde la corona sobre implante del 44, 45 y 46 inclusive hasta la zona retromolar. Mide aproximadamente $4 \mathrm{~cm}$ de longitud, con bordes difusos y eritematosos. 


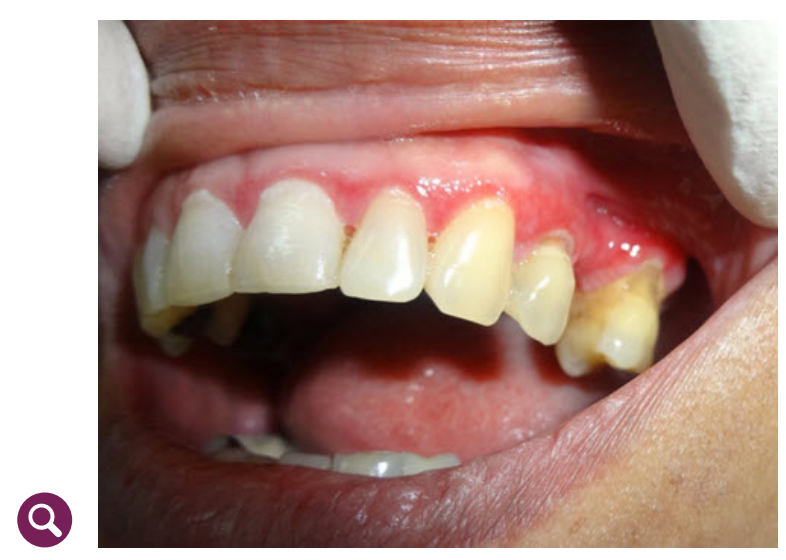

Figura 4. Gingivitis marginal crónica que compromete la encía libre desde la pieza dentaria 21 hasta la 26 inclusive.

Como manifestaciones clínicas en otras partes del cuerpo, el dorso de la mano izquierda presentaba máculas circulares de coloración rosa pálido de aproximadamente $0,5 \mathrm{~cm}$ de diámetro, donde la paciente anteriormente tuvo una lesión similar a una quemadura. Presentaba lesiones de la misma índole en la palma de la mano, próximo al talón del pie izquierdo, en la región submamaria derecha y signos de alopecia (Figura $\underline{5}, \underline{6}$ y $\underline{7}$ ).

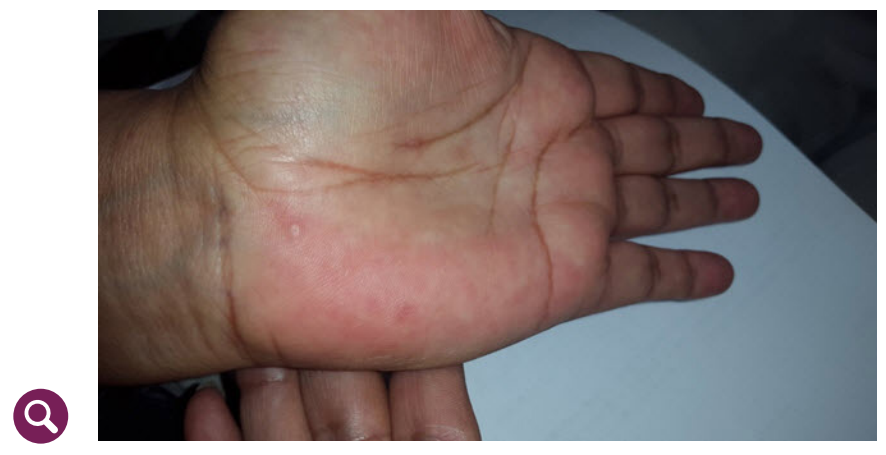

Figura 5. Se observa en la palma de la mano máculas circulares de coloración rosa pálido, de aproximadamente $0,5 \mathrm{~cm}$ de diámetro.
Q

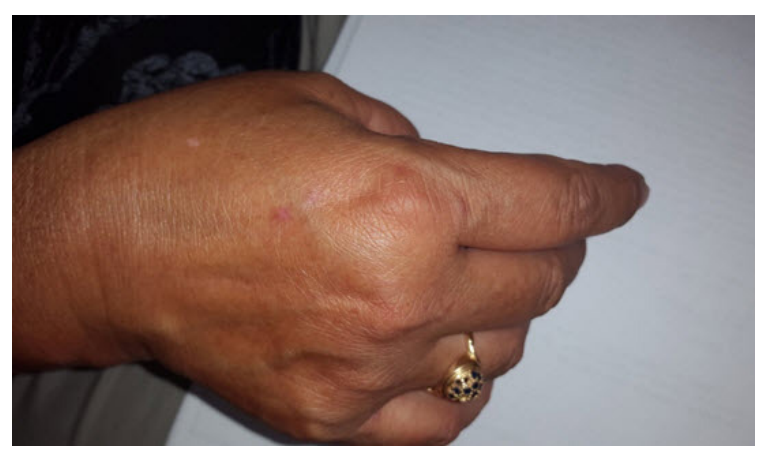

Figura 6. Se observan máculas circulares de coloración rosa pálido y blanquecinas de aproximadamente 6 meses de evolución

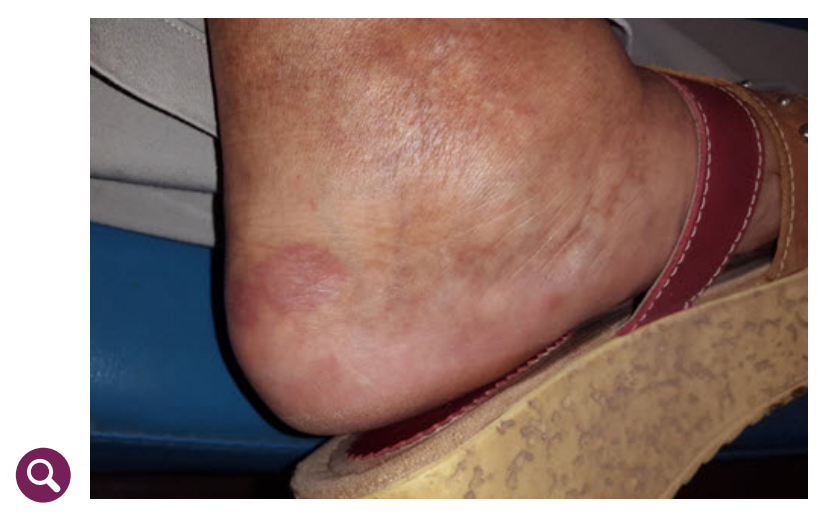

Figura 7. Se observa en la zona cutánea próxima al talón una lesión circular, rosa pálida, bien definida, sin relieve, de superficie lisa, de aproximadamente $1,5 \mathrm{~cm}$ de diámetro. Se acompaña de lesión circulares similares de menor tamaño de 3 meses de evolución. 
Se solicitaron exámenes complementarios como análisis de laboratorio y radiografía panorámica para observar el estado de los implantes dentarios colocados en boca en relación a las lesiones evidenciadas, así como posibles focos sépticos. En la primera cita, previo consentimiento informado, se le realizó una citología exfoliativa de las lesiones de fondo de surco vestibular derecho e izquierdo obteniendo como resultado candidiasis. Se procedió a realizar una biopsia de tipo incisional en las lesiones de la mucosa yugal del lado derecho y en fondo de surco vestibular derecho. Los fragmentos de tejido extraídos fueron introducidos en formol al 10\% y enviados al Servicio de Anatomía Patológica de la Facultad de Odontología con su correspondiente protocolo anatomopatológico. Las secciones mostraron dos fragmentos, uno correspondiente a mucosa constituida por epitelio plano estratificado no queratinizado, con acantosis irregular, exocitosis de células mononucleares y espongiosis. La misma no presentaba corion. El fragmento restante correspondia a tejido conectivo edematoso con escaso infiltrado mononuclear sin revestimiento epitelial. Se obtuvieron hallazgos vinculables a liquen ampollar (Figura 8).

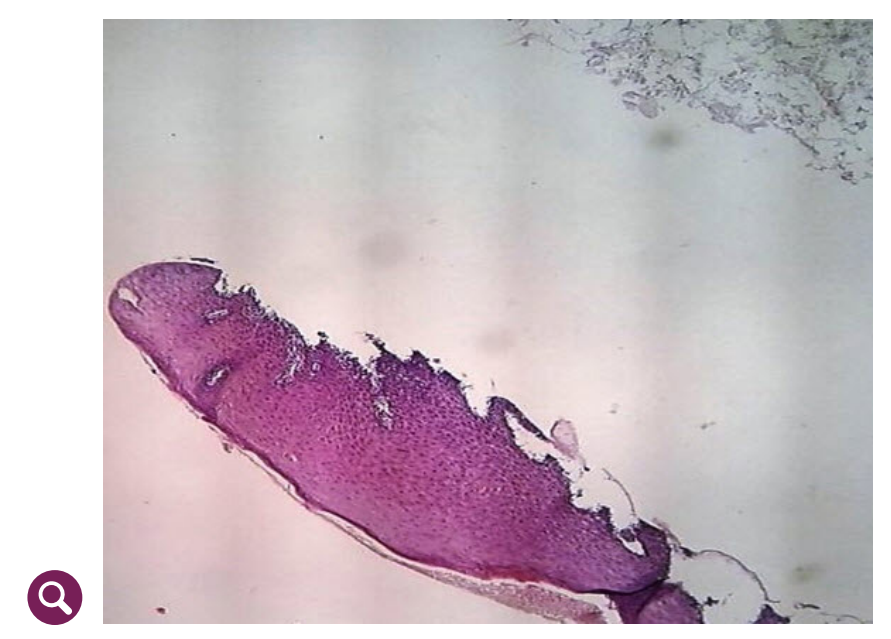

Figura 8. Prueba H/E: Se observa mucosa constituida por epitelio plano estratificado no queratinizado, con acantosis irregular, exocitosis de mononucleares y espongiosis; la misma no presenta corion. El fragmento restante corresponde a tejido conectivo edematoso con escaso infiltrado mononuclear sin revestimiento epitelial.

Para su tratamiento, se prescribió acetónido de triamcinolona, nistatina, vitamina $A$, Ácido Retinoico y Orabase ${ }^{\circledR}$ (Colgate-Palmolive Co., New York) en ungüento, aplicado tres veces por día durante diez días. Se enfatizó sobre técnica de cepillado para mejorar su calidad de higiene oral. Se recomendó que el tratamiento fuese multidisciplinario en conjunto con el dermatólogo, médico clínico y psicoanalista, por lo que fue derivada a consulta de Psicología en nuestra institución para ayudar a enfrentar este tipo de patología y su origen. A los diez días la paciente asistió al control observándose tejidos blandos en buen estado con disminución de la sintomatología. Se aconsejó continuar con la medicación descrita hasta la remisión completa de las lesiones y la eliminación de las coronas implanto-soportadas que estuvieran ocasionando traumas. Se procederá a la rehabilitación bucal integral una vez que desaparezcan los signos clínicos de la enfermedad.

\section{Discusión}

El liquen plano ampollar es una enfermedad inflamatoria mucocutánea crónica con fisiopatología inmune (10). Según Rodríguez-Calzadilla, el liquen ampollar es poco 
frecuente encontrarlo en boca (11). En lo que respecta a nuestra experiencia se coincide con esta afirmación debido a la escasez de casos clínicos con este diagnóstico definitivo y en relacion a la forma de presentación muco-cutánea de la enfermedad, siendo está benigna y crónica.

El presente caso clínico de liquen ampollar en una mujer de 60 años, se encuentra entre el grupo poblacional más prevalente (sexo femenino entre 30 y 70 años de edad) según Bascones-llundain y cols. (10). Las zonas topográficas de la cavidad bucal más afectadas según Márquez-Filiu y cols son: lengua, encía, mucosa yugal y surco vestibular. Es decir, todas aquellas zonas sometidas a roce (12). Se coincide con el caso clínico presentado donde las regiones más afectadas fueron los surcos vestibulares superior e inferior, la lengua, la mucosa yugal y las encías. Blanco-Carrión y cols. afirman que la localización típica del liquen ampollar es la mucosa vestibular adyacente a los últimos molares (4). Este tipo de lesiones corresponden a zonas erosionadas de poca profundidad, producto de la ruptura de las ampollas. En los pacientes se producen síntomas como: glosodinia, glosopirosis, disfagia y odinofagia, entre otras (12), como lo relataba la paciente del caso clínico expuesto.

En una revisión sistemática sobre el tratamiento de esta patología se determinó que el propionato de clobetasol al 0,025\% administrado como aerosol alivió significativamente el dolor en comparación con la pomada. En otro estudio, se observó una diferencia significativa en el que favoreció a la solución de ciclosporina sobre el acetónido de triamcinolona al $0,1 \%$ en orabase. Por otro lado, se estudió el gel de áloe vera obteniéndose seis veces más probabilidades de dar lugar a una mejoría de al menos el $50 \%$ en los síntomas de dolor en comparación con el placebo (13). En lo que respecta al caso clínico presentado, el acétonido de triamcinolona al $0,1 \%$ en conjunto con la nistatina y otros componentes en menor porcentaje ofrecieron excelentes resultados.

\section{Conclusión}

Los profesionales de la salud tenemos la responsabilidad de acompañar e informar oportunamente a los pacientes portadores de lesiones compatibles con un liquen plano ampollar sobre su diagnóstico de manera precoz y diferencial, el origen y la evolución de esta enfermedad, así como sobre su tratamiento oportuno para evitar su posible malignización. El tratamiento odontológico oportuno comprendido entre profilaxis y prevención de la salud bucal, así como el tratamiento llevado a cabo por las demás especialidades le permitirán mejorar su calidad de vida.

\section{Referncias bibliográficas}

1. Hernández-García SH, Pratt-Ribet I, Rodríguez-Arencibia MA, Rodríguez-Hernández MA. Liquen plano oral versus gingivoestomatitis herpética aguda. Ciencias Médicas [Internet]. 2013 [citado 2016 Dic 05];17(6). Disponible en: http://scielo.sld. cu/scielo.php?script=sci arttext\&pid=S1561-31942013000600018

2. Bascones-Ilundain C, González Moles MA, Carrillo de Albornoz A, Bascones-Martínez A. Liquen plano oral (I): Aspectos clínicos, etiopatogénicos y epidemiológicos. Av Odontoestomatol [Internet]. 2006 Feb [citado 2016 Dic 05] ;22( 1 ): 11-19. Disponible en: http://scielo.isciii.es/scielo.php?script=sci_arttext\&pid=S0213-12852006001100002\&lng=es.

3. Colonia A, Vélez LF, Liquen Plano oral. CES Odontologia [Internet]. 2011 [citado 2016 Dec 05]; 24(2). Disponible en: http://revistas.ces.edu.co/index.php/odontologia/ article/view/1979/1379 
4. Blanco Carrión A, Otero Rey E, Peñamaría Mallón M, Diniz Freitas M. Diagnóstico del liquen plano oral. Av Odontoestomatol [Internet]. 2008 [citado 2016 Dec 05]; 24(1):11-31. Disponible en: http://scielo.isciii.es/pdf/odonto/v24n1/original1.pdf

5. Torrente-Castells E, Figueiredo R, Berini-Aytés L, Gay-Escoda C. Clinical features of oral lichen planus. A retrospective study of 65 cases. Med Oral Patol Oral Cir Bucal. [Internet]. 2010 [citado 2016 Dec 05]; 15(5):685-690. Disponible en: https:// www.ncbi.nlm.nih.gov/pubmed/20383118

6. Crincoli V, Di Bisceglie MB, Scivetti M, Lucchese A, Tecco S, Festa F. Oral lichen planus: update on etiopathogenesis, diagnosis and treatment. Immunopharmacol Immunotoxicol. [Internet]. 2011 [citado 2016Dec 05]; 33(1):11-20. Disponible en: https://www.ncbi.nlm.nih.gov/pubmed/20604639

7. Lanfranchi HE, Aguas SC, Sano SM. Transformación maligna del Liquen Plano Bucal atípico: Análisis de 32 casos. Medicina Oral [Internet]. 2003 [citado 2016 Dec 05] ; 8:2-9. Disponible en: http://www.medicinaoral.com/medoralfree/v8i1/medoralv8i1p2.pdf

8. Carrozzo M, Thorpe R. Oral lichen planus: a review. Minerva Stomatol. [Internet]. 2009 [citado 2016 Dec 05]; 58(10):519-537. Disponible en: http://europepmc.org/ abstract/MED/19893476.

9. Colonia A, Vélez LF. Liquen Plano Oral. CES odontol. [Internet]. 2011 July [citado 2016 Dec 05] ; 24(2): 79-86. Disponible en: http://www.scielo.org.co/scielo. php?script=sci arttext\&pid=S0120-971X2011000200009\&lng=en.

10. Bascones-Ilundain C, González Moles MA, Carrillo de Albornoz A, Bascones-Martínez A. Liquen plano oral (I). Aspectos clínicos, etiopatogénicos y epidemiológicos. Av. Odontoestomatol [Internet]. 2006; [citado 2016 Dic 05] ; 22(1): 11-19. Disponible en: http://scielo.isciii.es/pdf/odonto/v22n1/original1.pdf

11. Rodríguez Calzadilla OL. Manifestaciones mucocutáneas del liquen plano: Revisión bibliográfica. Rev Cubana Estomatol [Internet]. 2002 Ago [citado 2016 Dic 05] ; 39(2): 157-186. Disponible en: http://scielo.sld.cu/scielo.php?script=sci_arttext\&pid=S0034-75072002000200006\&lng=es.

12. Márquez Filiu M, Estrada Pereira GA, González Heredia E, Medina Montoya LT, Jaca Portuondo AL. Manifestaciones bucales del liquen plano. MEDISAN [Internet]. 2013 Nov [citado 2016 Dic 05]; 17(11): 8023-8030. Disponible en: http:// scielo.sld.cu/scielo.php?script=sci_arttext\&pid=S1029-30192013001100007\&lng=es.

13. Cheng S, Kirtschig G, Cooper S, Thornhill M y cols. Interventions for erosive lichen planus affecting mucosal sites. Cochrane Database of Systematic Reviews [Internet]. 2012 [citado 2016 Dic 05]; Issue 6. Disponible en: https://www.ncbi.nlm.nih. gov/pubmed/22336835 